\title{
Optimization of the Phase-Locked Flux-Flow Oscillator for the Submm Integrated Receiver
}

\author{
Valery P. Koshelets, Pavel N. Dmitriev, Andrey B. Ermakov, Alexander S. Sobolev, \\ Mikhail Yu. Torgashin, Vladislav V. Kurin, Andrey L. Pankratov, and Jesper Mygind
}

\begin{abstract}
The Superconducting Integrated Receiver (SIR) comprises in one chip a planar antenna integrated with an SIS mixer, a superconducting Flux Flow Oscillator (FFO) acting as Local Oscillator (LO) and a second SIS harmonic mixer (HM) for FFO phase locking. Free-running FFO linewidth well below $10 \mathrm{MHz}$ is required to ensure phase-locked operation of an SIR. Comprehensive experimental study of the Nb-AlOx-Nb FFO linewidth and other main parameters has been carried out in order to achieve this goal. Essential dependence of the FFO linewidth on its width and idle region dimension has been found. It makes possible an optimization of the FFO design and selection of the best FFO parameters for practical operation of the SIR.
\end{abstract}

Index Terms - Josephson junctions, phase-locked oscillators, submillimeter wave integrated circuits, superconducting devices.

\section{INTRODUCTION}

A Josephson Flux Flow Oscillator (FFO) [1] has proven to be the most developed superconducting local oscillator for integration with an SIS mixer in a single-chip submmwave Superconducting Integrated Receiver (SIR) [2]. Such a receiver comprises in one chip a planar antenna and an SIS mixer, pumped by an integrated FFO. In order to obtain the frequency resolution required for practical application of a heterodyne spectrometer (of at least one part per million) the integrated local oscillator (LO) must be phase-locked to an external reference. To achieve this goal a concept of the integrated receiver with phase-locked loop (PLL) has been developed [3], [4]. Following this concept a $350 \mathrm{GHz}$ singlechip receiver containing a quasioptical SIS mixer, a flux flow

Manuscript received October 4, 2004.

The work was supported in parts by the RFBR projects 03-02-16748, 0202-16775; INTAS project 01-0367, ISTC project \# 2445, and the President Grant for the Scientific School 1344.2003.2, the RSSF and the Hartmann Foundation.

V. P. Koshelets, P. N. Dmitriev, A. B. Ermakov, A. S. Sobolev, and M. Yu. Torgashin are with the Institute of Radio Engineering and Electronics, Russian Academy of Science, Mokhovaya 11, 125009, Moscow, Russia; they are partially with the Space Research Organization of the Netherlands (SRON), P.O. Box 800, 9700 AV Groningen, the Netherlands, (telephone: 7095-2032784, e-mail: valery@hitech.cplire.ru)

V. V. Kurin, and A. L. Pankratov are with the Institute for Physics of Microstructure, Russian Academy of Sciences, GSP-105, 603950, Nizhny Novgorod, Russia (telephone: 7-8312-675185, e-mail: alp@ipm.sci-nnov.ru).

J. Mygind is with the Department of Physics, Technical University of Denmark, B 309, DK-2800 Lyngby, Denmark, (telephone: 45-45-253268, email: myg@fysik.dtu.dk) oscillator and a harmonic SIS mixer (HM) for FFO phase locking has been designed, fabricated and successfully tested [5], [6], showing the frequency resolution of the receiver as good as $10 \mathrm{kHz}$. The effect of broadening of a spectral line of $\mathrm{SO}_{2}$ gas at $326.867 \mathrm{GHz}$ was measured by this spectrometer for a laboratory gas cell within the pressure range of 0.030.3 mbar demonstrating the feasibility of such device for practical applications [6]. These achievements enabled the development of a $600-650 \mathrm{GHz}$ integrated receiver for the Terahertz Limb Sounder (TELIS) [7] intended for atmosphere study and scheduled to fly on a balloon in 2006.

There is a number of important requirements on the FFO properties to make it suitable for application in the phaselocked SIR. Obviously the FFO should emit enough power to pump an SIS mixer (taking into account a specially designed mismatch of about 5-7 dB between the FFO and the SIS mixer, introduced to avoid leakage of the input signal to the LO path). Even for ultra wideband PLL system an effective regulation bandwidth is limited by length of the cables (about $10 \mathrm{MHz}$ for typical loop length of two meters). It means that free-running FFO linewidth has to be well below $10 \mathrm{MHz}$ to ensure stable FFO phase locking with reasonably good spectral ratio (SR) - the ratio between the carrier and total power emitted by FFO [8]. For example, only about $50 \%$ of the FFO power can be phase-locked by the present PLL system at free-running FFO linewidth of $7 \mathrm{MHz}$. Low spectral ratio results in considerable error at resolving of the complicated line shape [7]. Thus sufficiently small freerunning FFO linewidth is vitally important for realization of the phase-locked SIR for TELIS.

Detailed measurements of the FFO linewidth [5], [8] demonstrate Lorentzian shape of the FFO line in a wide frequency range up to $700 \mathrm{GHz}$, both at higher voltages on the flux flow step (FFS) and at lower voltages in the resonant regime on the Fiske steps (FS's). It means that the freerunning ("natural") FFO linewidth in all operational regimes is determined by the wideband thermal fluctuations and the shot noise. This is different from many traditional microwave oscillators where the "natural" linewidth is very small and the observed linewidth can be attributed mainly to external fluctuations. It was found [5], [8], [9] that free-running FFO linewidth, $\delta f$, exceed theoretical estimations made for lumped tunnel Josephson junction. To explain the experimentally measured dependence of the FFO linewidth, an additional term proportional to differential resistance on control line 
current, $R_{d}^{C L}$, (control line produces magnetic field for FFO operation) has been introduced to the noise model of FFO [5], [8], [10]: $\delta f=\left(2 \pi / \Phi_{0}^{2}\right)\left(R_{d}{ }^{B}+K^{*} R_{d}^{C L}\right)^{2} S_{i}(0)$; where $S_{i}(0)$ is the power density of low frequency current fluctuations. This term can be explained by an assumption that a part of the DC bias current creates an additional magnetic field in the junction [8], [11].

Previous linewidth measurements have demonstrated [10], essential dependence of the free-running FFO linewidth on the FFO voltage, its current density and geometry of the biasing electrodes. In this report some results of the further FFO study and optimization of the FFO layout are presented. For these measurements we used the integrated circuits [4] comprising FFO and harmonic mixer $\mathrm{HM}$ with matching circuits for linewidth measurements and FFO phase locking.

\section{DEPENDENCE OF THE FFO PROPERTIES ON ITS PARAMETERS}

\section{A. FFO power detected by HM.}

The FFO is a long Josephson junction of the overlap geometry; the following parameters are used in this study $\mathrm{J}_{\mathrm{c}}=$ $7 \mathrm{kA} / \mathrm{cm}^{2}, \mathrm{RnS}=30 \Omega^{*} \mu \mathrm{m}^{2}$, length, $\mathrm{L}=400 \mu \mathrm{m}$, width, $\mathrm{W}=$ 4-16 $\mu \mathrm{m}$, overlapping of the electrodes (width of the idle region on each side of the FFO), $\mathrm{WI}=2-10 \mu \mathrm{m}$. A family of the FFO IVCs measured at different magnetic fields produced by the integrated control line is presented in Fig. 1a ( $\mathrm{W}=$ $8 \mu \mathrm{m}, \mathrm{WI}=10 \mu \mathrm{m})$. Single SIS junction with inductive tuning circuit is employed as HM. The tuning and matching circuits were designed to provide "uniform" coupling in the frequency range $400-700 \mathrm{GHz}$. Measured value of the HM current induced by the FFO oscillations (HM pumping) is shown in Fig. 1a by the color scale. Maximum of the HM pumping for each FFO frequency measured at constant HM bias voltage $2.5 \mathrm{mV}$ (normalized on the current jump at the gap voltage, $\mathrm{I}_{\mathrm{g}}$ $=115 \mu \mathrm{A}$ ) is presented in Fig. $1 \mathrm{~b}$. From Fig. 1 one can see that an FFO can provide large enough power over the wide frequency range limited only by $\mathrm{Nb}$ superconducting gap.

An important issue for TELIS operation is a possibility to tune the FFO frequency and power independently providing the same spectral ratio of PL FFO. The SR value for the given PLL system is determined by free-running FFO linewidth: these two quantities are unambiguously related (see Fig. 2 where data for FFO of different designs are presented). Theoretical curve, calculated for the simplest first order PLL model, coincides reasonably with experimental data.

Linewidth and SR for the FFO of TELIS design are almost constant over very wide range of FFO bias current at fixed FFO frequency (see Fig. 3). From this figure one can see that the SR is of about $50 \%$ over the range of bias current, $\mathrm{I}_{\mathrm{b}}, 14-$ $30 \mathrm{~mA}$, while the pumping level varies from $3.5 \mu \mathrm{A}$ at $\mathrm{I}_{\mathrm{b}}=$ $14 \mathrm{~mA}$ up to $81 \mu \mathrm{A}$ at $\mathrm{I}_{\mathrm{b}}=30 \mathrm{~mA}$; furthermore $\mathrm{SR}=34 \%$ can be realized at $\mathrm{I}_{\mathrm{b}}=12 \mathrm{~mA}$ where the HM pumping is below $0.5 \mu \mathrm{A}$. It means that the HM operates in highly non-linear regime and even moderate HM pumping is enough for efficient PLL operation ensuring large enough signal to noise ratio.
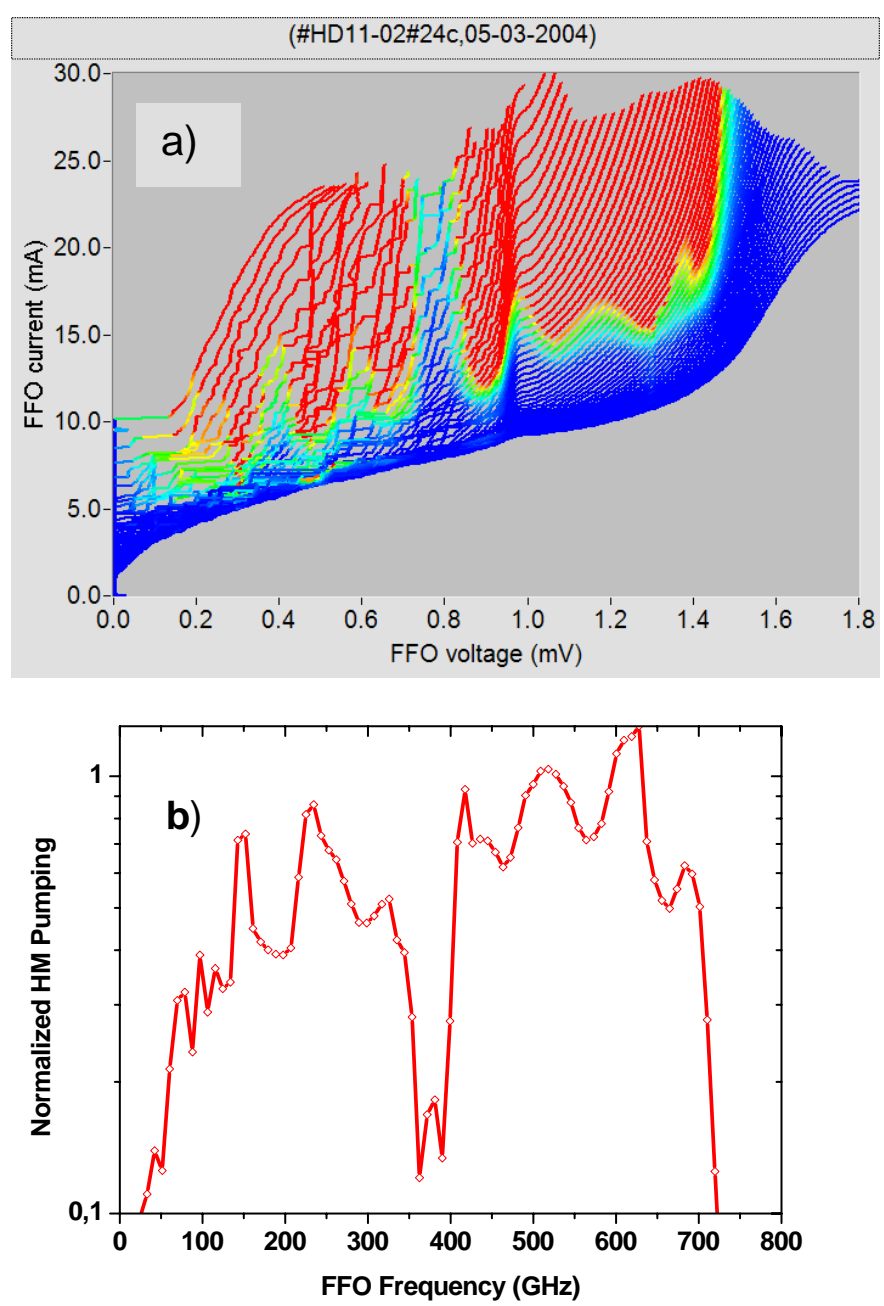

Fig. 1. a) IVCs of the FFO of a new design measured at different magnetic fields produced by the integrated control line. The tone scale shows the level of the DC current rise at HM induced by FFO. Black area marks the region of the FFO parameters where the induced by FFO HM current exceeds $25 \%$ of the $I_{\mathrm{g}}$. This level is well above the optimal value for an SIS-mixer operation. b) Maximum current detected by $\mathrm{HM}$ at $\mathrm{V}=2.5 \mathrm{mV}$ normalized on the current rise at the HM gap voltage, $\mathrm{I}_{\mathrm{g}}\left(\mathrm{I}_{\mathrm{g}}=115 \mu \mathrm{A}\right)$ as a function of the FFO frequency. Frequency scale of Fig. 1b corresponds to voltage scale of the Fig. 1a according to Josephson relation. Note that the dip around $370 \mathrm{GHz}$ is due to design of the single-junction HM and exactly corresponds to numerical calculations.

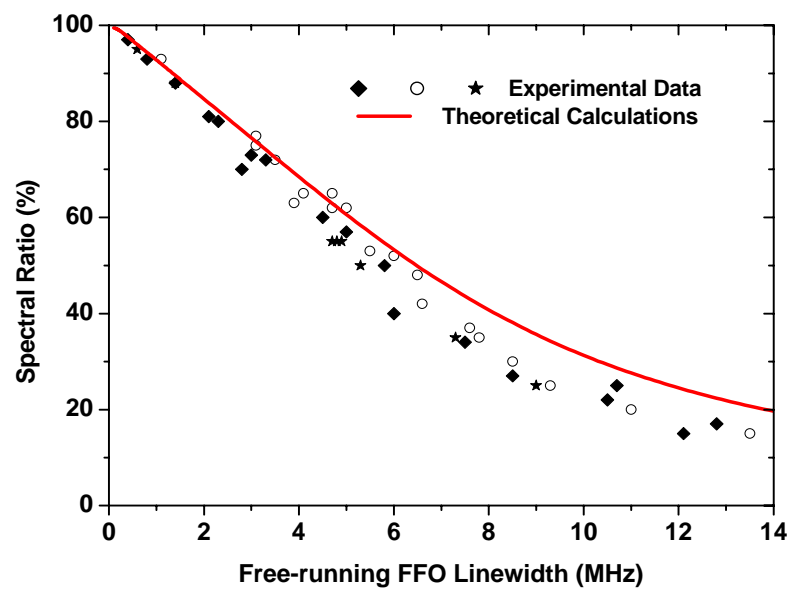

Fig. 2. Spectral Ratio for the phase-locked FFO of different design as a function of free-running FFO linewidth. 
B. FFO linewidth: dependence on the FFO frequency and current density.

Dependences of the FFO linewidth and SR of the phaselocked FFO on the FFO frequency measured at constant bias current $\mathrm{I}_{\mathrm{b}}=21 \mathrm{~mA}$ are presented in Fig. 4. Abrupt increase of the linewidth at the FFO frequency of about $450 \mathrm{GHz}$ is caused by the effect of Josephson self-coupling (JSC) [12]. The JSC considerably modifies the FFO properties at voltages $\mathrm{V} \approx \mathrm{V}_{\mathrm{JSC}}=1 / 3 * \mathrm{~V}_{\text {gap }}\left(\mathrm{V}_{\text {JSC }}\right.$ corresponds to $450 \mathrm{GHz}$ for a Nb$\mathrm{AlO}_{\mathrm{x}}-\mathrm{Nb} \mathrm{FFO}$ ) and results in larger internal damping at voltages $\mathrm{V}>\mathrm{V}_{\text {JSC }}$ that significantly complicates phase locking of the FFO.

For all tested samples there is a well-defined dependence of the free-running FFO linewidth on frequency at voltages $\mathrm{V}>$ $\mathrm{V}_{\text {JSC }}$ (see Fig. 4). Since the data are taken at a constant bias current and a voltage dependence of the FFO linewidth is negligibly small, the variation of the linewidth on the bias voltage can be mainly attributed to changes in the differential resistance. Presumably the dependence of the $\mathrm{R}_{d}$ and $\mathrm{R}_{d}{ }^{\mathrm{CL}}$ on FFO frequency (voltage) reflects the fact that introduction of an additional fluxon into the junction requires a larger control line current as the fluxon chain gets denser. This assumption was preliminary confirmed by numerical calculations [10].

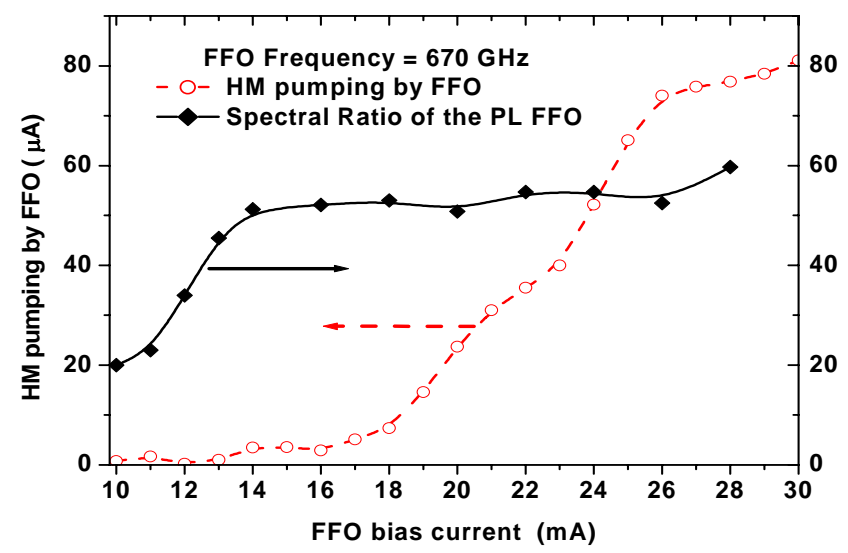

Fig. 3. Dependence of the HM current induced by FFO (HM pumping) and Spectral Ratio after FFO phase locking as a function of FFO bias current. All data measured at FFO frequency of $670 \mathrm{GHz}$.

Previous measurements [10] demonstrate also a considerable increase of the FFO linewidth with FFO current density. It is in contradiction with simplified consideration: the increase of the FFO current density should result in the increase of the total FFO bias current and in the decrease of the FFO differential resistance. Since the FFO linewidth is proportional to $\mathrm{R}_{\mathrm{d}}{ }^{2} * \mathrm{I}_{\mathrm{b}}$, the increase of the FFO current density has to be accompanied by the decrease of the measured FFO free-running linewidth. In reality the increase of the linewidth is caused by the fact that decrease of the differential resistance is smaller than expected. It may be explained by larger influence of the JSC effect and/or by more inhomogeneous bias current distribution for larger $\mathrm{J}_{\mathrm{c}}$ ). Nevertheless the linewidth can be quantitatively calculated from the developed FFO model [8], [11] using the experimentally measured parameters. It proves that there is no additional broadening, although there is still a lack of a complete FFO model, capable of predicting differential resistances. High value of the current density $\left(\mathrm{J}_{\mathrm{c}} \geq 8 \mathrm{kA} / \mathrm{cm}^{2}\right)$ is important for wide-band operation of an SIS mixer at submm wave range. The discussed above increase of the FFO linewidth with current density creates serious problem in design and development of SIR chips. Implementation of two separate trilayes with different current densities - one for the SIS mixer (high $\mathrm{J}_{\mathrm{c}}$ ) and the other for the FFO (lower $\mathrm{J}_{\mathrm{c}}$ ) might be a solution.

\section{FFO linewidth: dependence on the FFO width and its geometry.}

To study the dependence of the FFO linewidth on its width, $\mathrm{W}$, a number of FFOs with the same electrodes layout, but different width of FFO junction ( $\mathrm{W}=4,8,12$ and $16 \mu \mathrm{m}$ ) were fabricated in one technological batch $\left(\mathrm{RnS}=30 \Omega^{*} \mu \mathrm{m}^{2}\right)$. The results of the LW measurements of these circuits at three FFO frequencies are presented in Fig. 5. One can see that for all frequencies of interest FFO linewidth considerably decreases with increasing of the FFO width (consequently, the spectral ratio is getting much higher). Furthermore, there is no visible saturation of this effect in the studied range of the FFO width except for FFO frequency $706 \mathrm{GHz}$, where there is some saturation of SR, but presumably it is rather due to PLL system operation than FFO properties.

In principle such behavior is quite explainable according to mentioned above simplified model: FFO linewidth, proportional to $\mathrm{R}_{\mathrm{d}}{ }^{2} \mathrm{I}_{\mathrm{b}}$, has to go down with increase of the FFO width since it should result in increase of total FFO bias current and in decrease of the FFO differential resistance. Fortunately, in contrast to the case of changing of the FFO current density, the $R_{d}$ value indeed decreases more or less inversely proportional to $I_{b}$. Furthermore, it seems that $R_{d}^{C L}$ value decreases even stronger than $R_{d}$, especially at FFO frequencies of about $500 \mathrm{GHz}$ just above $\mathrm{V}_{\text {JSC }}$. Of course, one can expect saturation of the linewidth decrease and deterioration of the FFO behavior at further width increase (for example, due to appearance of the transversal modes). Since there is no reliable theory the optimal value of the FFO width has to be determined experimentally.

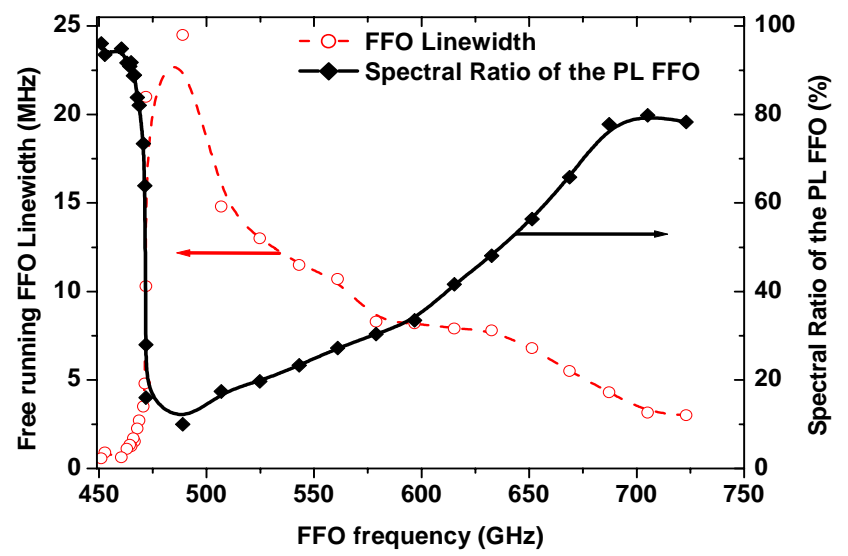

Fig. 4. FFO linewidth and Spectral Ratio of the phase-locked FFO on its oscillation frequency. The data are measured at constant bias current, while the FFO frequency is tuned by the magnetic field (by the control line current). 


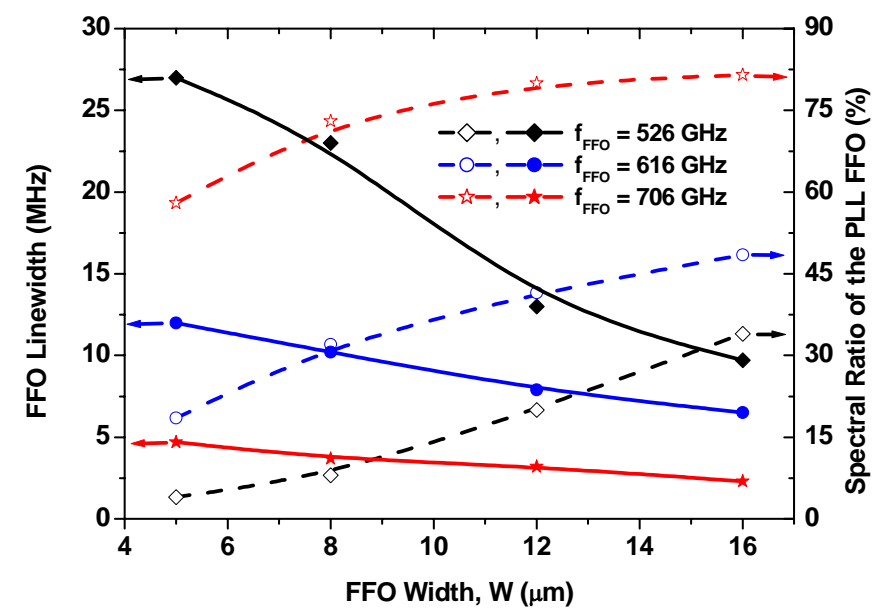

Fig. 5. Linewidth of free-running FFOs (left axis) and according Spectral Ratio for the phase-locked FFO (right axis) measured at different FFO frequencies as a function of FFO width. All circuits are taken from the same batch, $\mathrm{RnS}=30 \mathrm{Ohm}^{*} \mu \mathrm{m}^{2}$.

Even better performance was obtained for FFO of a little different design with enlarged electrodes overlapping (Fig. 6). Larger overlapping presumably provides more uniform bias current distribution - due to much smaller inductance of the overlapping electrodes. Larger overlapping of the FFO electrodes means also that the FFO of the same width is shifted from the edge of the bottom electrode, that results in considerable decrease of the $\mathrm{R}_{\mathrm{d}}{ }^{\mathrm{CL}}$ value. Note that for wide FFO some shift of the FFO centerline appears "automatically" due to increasing of the width. Up to now there is no adequate model that can describe quantitatively both the processes in the FFO and a self-consistent distribution of the bias current. Nevertheless, presented results are very encouraging and these modifications of the FFO can be recommended for implementation in the TELIS SIRs.

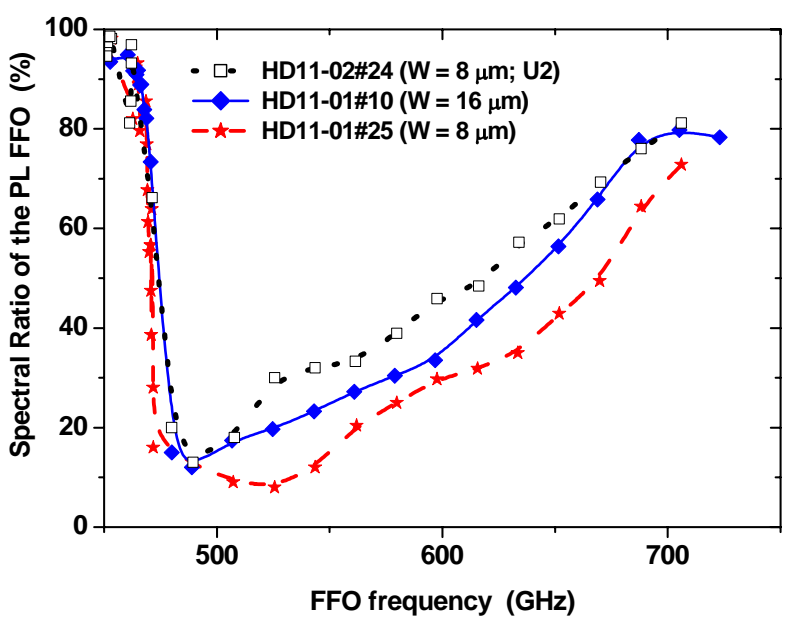

Fig. 6. Spectral Ratio of the phase-locked FFO of a new design with increased overlapping of the electrodes (U2) as a function of FFO frequency. Data for the FFOs of different width with smaller overlapping are shown for comparison. All circuits are from the same batch, $\mathrm{RnS}=30 \mathrm{Ohm}^{*} \mu \mathrm{m}^{2}$.
To explore further this approach we developed different designs of the "self-shielded" FFO with large ground plane. Such FFO supposed to be much more protected from variations of external magnetic field and has to provide much more uniform bias current distribution (since all bias leads are laying over superconducting shield and have very low inductance). Actually, low inductive bias leads provide a possibility of optimal (rather than uniform) current distribution, "requested" by the FFO itself. The last feature must ensure maximum of emitted FFO power. Indeed, the IVCs of all shielded FFOs are much more reproducible; the power, delivered to HM is higher compared to a "standard" design (for the same FFO width and for the same design of HM and matching circuits). Unfortunately, free-running linewidth for all variants of shielded FFO is much larger than linewidth for FFO of traditional design. It seems that injection of the bias via separate leads results in some spatial modulation of bias current [10] despite the additional triangular elements added for more uniform current injection.

In conclusion, improved design of the FFO for TELIS has been developed and optimized. Free-running linewidth value from 9 to $2 \mathrm{MHz}$ has been measured in the frequency range $500-710 \mathrm{GHz}$. As a result the spectral ratio of the phasedlocked FFO varies from 35 to $87 \%$ correspondingly, ensure at least of half phase-locked FFO power in the primary frequency range for TELIS 600-650 GHz.

\section{REFERENCES}

[1] T. Nagatsuma, K. Enpuku, F. Irie, and K. Yoshida, "Flux-flow type Josephson oscillator for millimeter and submillimeter wave region," $J$. Appl. Phys., vol. 54, p. 3302, 1983, see also Pt. II: J. Appl. Phys. Vol. 56, p. 3284, 1984; Pt. III, J. Appl. Phys., vol. 58, p. 441, 1985; Pt. IV, J. Appl. Phys., vol. 63, p. 1130, 1988.

[2] V. P. Koshelets and S. V. Shitov, "Integrated Superconducting Receivers," Superconductor Science and Technology, vol. 13, pp. R53R69, 2000.

[3] V. P. Koshelets et al., "Flux Flow Oscillators for Sub-mm Wave Integrated Receivers," IEEE Trans. on Appl. Supercond., vol. 9, no. 2, pp. 4133-4136, 1999.

[4] V. P. Koshelets et al, " Phase Locking of 270-440 GHz Josephson Flux Flow Oscillator," Rev of Sci Instr., vol. 71, pp. 289-293, 2000.

[5] V. P. Koshelets et al., "Towards a Phase-Locked Superconducting Integrated Receiver: Prospects and Limitations,” Physica C, vol. 367, pp. 249-255, 2002.

[6] S. V. Shitov et al., "An Integrated Receiver with Phase-Locked Superconducting Oscillator," IEEE Trans. on Appl. Supercond.y, vol. 13-2, pp. 684-687, June 2003.

[7] V. P. Koshelets et al., "Superconducting Integrated Receiver for TELIS”, report 2ET06, this conference.

[8] V.P. Koshelets et al., "Line width of Josephson flux flow oscillators", Physica C, vol. 372-376, p. 316-321, 2002

[9] V.P. Koshelets et al., "Externally Phase-Locked Local Oscillator for Submm Integrated Receivers: Achievements and Limitations", IEEE Trans. Appl. Supercond., vol. 13-2, p. 1035-1038, 2003.

[10] V. P. Koshelets et al., "Superconducting Phase-Locked Local Oscillator for Submm Integrated Receiver”, Supercond. Sci. Technol., vol. 17, p. 127-131, 2004

[11] A. L. Pankratov, "Form and width of spectral line of a Josephson flux flow oscillator", Phys. Rev. B, vol. 65, p. 054504-(1-9), 2002.

[12] V. P. Koshelets, S. V. Shitov, A. V. Shchukin, L. V. Filippenko, J. Mygind, and A. V. Ustinov, "Self-Pumping Effects and Radiation Linewidth of Josephson Flux Flow Oscillators", Phys Rev B, vol. 56, p. 5572-5577, 1997. 